\title{
INTERPRETIVE SUMMARIES, APRIL 2012
}

Effect of temperature and pH on the solubility of caseins: Environmental influences on the dissociation of $\boldsymbol{\alpha}_{\mathrm{S}^{-}}$and $\boldsymbol{\beta}$-casein. By Post et al., page 1603. Selective precipitation is a common method for isolating food-grade $\beta$-casein based on the different calcium sensitivities of the individual caseins and the selective solubilization of $\beta$-casein at a low temperature. Yield of $\beta$-casein depends on the raw material. In this work, we evaluated the effect of temperature and $\mathrm{pH}$ on the solubility of $\alpha_{S^{-}}$and $\beta$-casein in solutions of different casein preparations. A simple solubility analysis may be used to estimate the yield of $\beta$-casein fractions so that suitable casein raw materials for isolation can be selected in advance.

Twelve-year cohort study on the influence of caprine arthritis-encephalitis virus infection on milk yield and composition. By Kaba et al., page 161\%. The study was based on a long (12 consecutive years) and detailed (3,042 records from 177 animals) observation of a dairy goat herd with a chronic, naturally occurring caprine arthritis-encephalitis virus (CAEV) infection. The goal of the study was to dispel existing doubts on the effect of CAEV infection on the milk production and quality of milk. The study showed that $\mathrm{CAEV}$ infection does not reduce milk yield or elevate SCC. Quality of milk is negatively affected by CAEV infection with respect to total protein, fat, and lactose.

Heat stability of milk supplemented with calcium chloride. By On-Nom et al., page 1623. The effects of addition of calcium chloride on the heat stability of milk subject to an in-container heating process between 60 and $120^{\circ} \mathrm{C}$ were studied. Samples were subjected to dialysis during heating, and both $\mathrm{pH}$ and ionic calcium decreased in dialysates as temperature increased. The transition conditions between good and poor heat stability were established at different calcium chloride concentrations and at different temperatures. As temperature increased, coagulation occurred at lower levels of added calcium chloride. At lower temperatures, the transition was less distinct, with an increase in sediment formation before a firm coagulum was formed.

Characterization of coagulase-positive staphylococci isolated from tank and silo ewe milk. By Linage et al., page 1639. Coagulase-positive staphylococci are important animal and human pathogens. This study focused on the characterization of staphylococci isolated from ewe milk and the study of the prevalence of potentially enterotoxigenic strains. Staphylococcus aureus isolates carrying enterotoxin $\mathrm{C}$ gene were dominant among the population of coagulase-positive staphylococci isolated from ewe milk. No methicillinresistant isolates were detected. These results can help to improve the microbiological safety of dairy products manufactured from raw milk.

Selection of potential probiotic lactobacilli for cholesterol-lowering properties and their effect on cholesterol metabolism in rats fed on a high-lipid diet. By Wang et al., page 1645. The World Health Organization has predicted that by 2030, cardiovascular diseases will remain one of the leading causes of death, affecting approximately 23.6 million people worldwide. One of the major risk factors for coronary heart disease is hypercholesterolemia. Probiotics are live microbial feed supplements that beneficially affect the host animal by improving its intestinal microbial balance. One of the beneficial health effects related to probiotics is their ability to reduce serum cholesterol. The aim of this study was to screen lactobacilli with probiotic characteristics, and to determine and compare the effect of the screened Lactobacillus strains on cholesterol metabolism. The 3 screened Lactobacillus strains had strong bile salt resistance, low $\mathrm{pH}$ tolerance, and variant hypocholesterolemic properties in vitro, and were able to reduce cholesterol in vivo.

Functional properties of whey proteins microparticulated at low pH. By Dassanayake et al., page 166\%. Heat denaturation of whey proteins (WP) will adversely affect their important functional properties such as colloidal stability and solubility, creating undesired aggregates and limiting their application in food systems. At low $\mathrm{pH}$ and high hydrodynamic pressure, heat-denatured WP produce micro-aggregates with improved heat stability and emulsifying properties. Altering the $\mathrm{pH}$ resulted in modulation of intra- and intermolecular protein interactions that led to substantial reductions in particle size in microparticulated WP, creating an opportunity to further diversify application of this important dairy ingredient.

Optimal growth of Lactobacillus casei in a Cheddar cheese ripening model system requires exogenous fatty acids. By Tan et al., page 1680. The nature of cheese makes it difficult to study the complex microbial and biochemical processes that result in the development of Cheddar cheese flavor. Therefore, a Cheddar cheese model system, based upon an aqueous extract of Cheddar cheese, was developed to allow for the systematic study of these processes. This study reports an improvement to the model system that allows for growth of microorganisms, known to contribute to cheese flavor development, to typical levels.

Consumer perception and sensory effect of oxidation in savory-flavored yogurt enriched with n-3 lipids. By Rognlien et al., page 1690. The sensory 
characteristics of a fish oil-enriched savory-flavored yogurt were examined using sensory evaluation. Effects of oxidation and flavor interactions were examined. Oxidized fish oil diminished the perception of lime and acidity flavors and high levels of fresh fish oil. A chile-lime-flavored yogurt delivering levels of n-3 fatty acids within the recommended daily values per serving of yogurt (145 mg of docosahexaenoic acid + eicosapentaenoic acid/170 g) was liked by $50 \%$ of consumers.

Comparison of estrone and 17 $\beta$-estradiol levels in commercial goat and cow milk. By Farlow et al., page 1699. Since estrogens have been linked to breast cancer, many individuals are concerned with the amount of estrogens they consume in their diet. A major source of estrogens is milk. Although cow milk is most commonly consumed within the United States, more individuals consume goat milk worldwide. In this study, we measured the amounts of estrogens in various milk products and showed that goat milk has lower combined levels of estrogens than cow milk.

Short communication: Factors affecting coagulation properties of Mediterranean buffalo milk. By Cecchinato et al., page 1709. Milk coagulation properties (MCP) are important for cheese production in buffalo. The primary MCP measurements are rennet coagulation time (RCT, min) and curd firmness after $30 \mathrm{~min}$ from rennet addition $\left(\mathrm{a}_{30}, \mathrm{~mm}\right)$. Technological properties of milk are the outcome of interactive effects involving several factors. This study showed that acidity is an important source of variation of RCT and $\mathrm{a}_{30}$, along with fat content for RCT and casein content for $\mathrm{a}_{30}$. Hence, these MCP might be used as indicator traits to improve technological properties of buffalo milk.

Mechanical properties of the bovine claw horn during lactation. By Winkler and Margerison, page 1714. Claw horn disorders are one of the main causes of lameness and subsequent poor welfare of dairy cows. Changes in the structural properties of bovine claw horn were assessed by applying mechanical property testing techniques and comparing these with claw horn haemorrhage and locomotion scoring. The resistance to mechanical puncture was significantly decreased in haemorrhaged sole horn and was also lower in the white line area of the claw compared with the sole.

Endocrine milieu and developmental dynamics of ovarian cysts and persistent follicles in postpartum dairy cows. By Roth et al., page 1729. Development of follicular pathologies in the early postpartum period is involved in reduced fertility of high-yielding dairy cows. We explored the endocrine milieu in both follicular fluid and plasma and the growth dynamics of various ovarian type of cysts and persistent follicles, with an emphasis on characterizing their formation before the appearance of pathological signs. The findings contribute to our understanding of the formation of follicular pathologies through the early postpartum period.

Automatic measurement of touch and release angles of the fetlock joint for lameness detection in dairy cattle using vision techniques. By Pluk et al., page 1738. A measurement system is described that automatically records joint angles in walking dairy cattle using image processing. Analysis of these variables showed significant differences in joint angles between the different lameness levels (gait scores), making them useful indicators for lameness. Classification results on a group level indicated $65.4 \%$ correct classification between the 3 classes of lameness. By monitoring the variance and changes of the joint angle variables in individual cows, changes in lameness level could be automatically detected in a very large percentage of the cows (83.3 and $76.2 \%$, respectively).

Blood immunometabolic indices and polymorphonuclear neutrophil function in peripartum dairy cows are altered by level of dietary energy prepartum. By Graugnard et al., page 1749. Dairy cows experience some degree of negative energy balance (NEB) and immunosuppression around parturition, making them vulnerable to metabolic and infectious diseases. Immunometabolic responses were evaluated around parturition in cows overfed a moderate-energy diet or fed a high-straw diet to meet but not greatly exceed energy requirements during the dry period. Overfed cows were in more positive energy balance prepartum but more negative energy balance in the first week postpartum. The prepartum concentration of insulin in energy-overfed cows was greater and negatively related with the phagocytosis capacity of polymorphonuclear neutrophils, suggesting a potential impairment of the immune response.

Alterations of fatty acid $\beta$-oxidation capability in the liver of ketotic cows. By Li et al., page 1759. As a metabolic disease, ketosis is closely associated with liver lipidosis. Although progress has been made in understanding the pathology and etiology of ketosis in dairy cows, the underlying mechanisms remain unclear. These disorders affect the health, well-being, productivity, and reproductive performance of cows. In this study, we investigated fatty acid metabolism in ketotic and nonketotic cows by evaluating the expression of fatty acid $\beta$-oxidation-related enzymes in specimens of liver tissue. Overall, fatty acid $\beta$-oxidation capability was altered in the liver of ketotic compared with nonketotic cows.

Association of plasma insulin concentration to fatty acid distribution between milk fat and 
membrane synthesis. By Mesilati-Stahy et al., page 176\%. The milk lipid fraction consists of approximately 98\% triglycerides and 2\% phospholipids. Phospholipids are health-promoting bioactive molecules; thus, understanding the mechanisms controlling the balance between triglycerides and phospholipids in milk could have nutritional and health implications. The aim was to study the connection between milk fat concentration and the composition of its lipid compartments: triglycerides and phospholipids. Because insulin and milk fat concentration are tightly associated, we retrospectively studied the fatty acid distribution in milk triglycerides and phospholipids in cows with different plasma insulin concentrations. We found that metabolic factors modulate fatty acid utilization for milk phospholipids and triglyceride.

Effect of endocervical inflammation on days to conception in dairy cows. By Deguillaume et al., page 17r6. Inflammation of the uterine mucosa a few weeks after calving is common in dairy cows and associated with impaired subsequent reproductive performance. Unlike endometritis, very little is known about endocervical inflammation. This paper reports a high prevalence of endocervical inflammation at the end of involution period, with an effect on time to conception. Moreover, endocervical inflammation can occur in the absence of endometrial inflammation. Postpartum cervicitis in dairy cows should thus be investigated in addition to endometritis because of its high prevalence and of its specific effect on hazard of pregnancy.

On-farm estimation of energy balance in dairy cows using only frequent body weight measurements and body condition score. By Thorup et al., page 1784. Precise estimates of dairy cow energy balance are important in managing health, reproduction, and feed. Energy balance of 76 cows was estimated using body weight (BW) automatically measured at milking and corrected for milk and gutfill, and body condition scores (BCS) assessed visually every $2 \mathrm{wk}$. Changes in corrected BW and BCS were used to calculate changes in energy balance. The method agreed with the traditional method based on feed intake, milk, and maintenance. For farmers, estimating energy balance for individual cows on-farm without needing feed intake (which is hard to measure in practice) would be a desirable management tool.

Low progesterone concentration during the development of the first follicular wave reduces pregnancy per insemination of lactating dairy cows. By Denicol et al., page 1794. Recently we demonstrated that cows induced to ovulate the first follicular wave produced embryos of poorer quality because of exposure to progesterone ( $\mathrm{P} 4)$ concentrations $<2 \mathrm{ng} /$
$\mathrm{mL}$ during follicle growth. In the current study, cows induced to ovulate the first follicular wave had reduced pregnancy compared with those induced to ovulate the first follicular wave and treated with exogenous $\mathrm{P} 4$ and those induced to ovulate the second follicular wave. Although reduced P4 concentration during follicular growth increased number of corpora lutea after AI, induced ovulation of the first follicular wave reduced pregnancy per insemination because of exposure to $\mathrm{P} 4$ concentration $<2 \mathrm{ng} / \mathrm{mL}$.

An experimental infection model to induce digital dermatitis infection in cattle. By Gomez et al., page 1821. A bovine digital dermatitis (DD) infection model was developed to provide a standard way for reproducing DD infections in individual cattle independent of external factors that could influence the course of disease. Four heifers were challenged with homogenates of epidermal skin biopsies from active DD lesions or cultures of Treponema species. Successful reproduction of acute DD lesions was observed, which may be used to evaluate interventions to control DD and study its pathogenesis in a controlled manner.

Prostaglandin $F_{2 \alpha}$ and gonadotropin-releasing hormone administration improve progesterone status, luteal number, and proportion of ovular and anovular dairy cows with corpora lutea before a timed artificial insemination program. $B y$ Stevenson et al., page 1831. Administration of prostaglandin $\mathrm{F}_{2 \alpha}\left(\mathrm{PGF}_{2 \alpha}\right) 3 \mathrm{~d}$ before gonadotropin-releasing hormone (PG-3-G) compared with 2 injections of $\mathrm{PGF}_{2 \alpha} 14 \mathrm{~d}$ apart (Presynch-10) increased the proportion of cows having elevated progesterone and at least 1 corpus luteum (CL), and the number of CL per cow at the time cows were enrolled in a timed artificial insemination (AI) program $10 \mathrm{~d}$ after the second or only $\mathrm{PGF}_{2 \alpha}$ injection. Seven days later, PG-3-G cows had greater concentrations of progesterone and more CL per cow. In this preliminary study, resulting pregnancies per AI were only numerically greater at d 32 and 60, but PG-3-G compared with Presych-10 presynchronized key follicular and luteal traits in both ovular and anovular cows.

Risk factors and effects of postpartum anovulation in dairy cows. By Dubuc et al., page 1845. This study investigated risk factors and effects of postpartum ovulatory status in dairy cows. Factors associated with early ovulation included parity group, season, and decreased lipid mobilization, whereas risk factors for prolonged anovulation included season, milk production, and cytological endometritis. Prolonged anovulation increased time to first insemination and time to pregnancy; this effect was dependent on parity group as it only occurred in older cows. 
Economic analysis of Mycobacterium avium subspecies paratuberculosis vaccines in dairy herds. By Cho et al., page 1855. Mycobacterium avium ssp. paratuberculosis (MAP) is the causative pathogen of Johne's disease, which has no fail-safe prevention or cure. This study investigates the epidemiological effects and economic values of hypothetical MAP vaccines in dairy herds. We created scenarios for the potential epidemiological effects of MAP vaccines and then estimated economically justifiable monetary values at which they would be cost-effective to dairy producers. The estimated economic values of MAP vaccines suggested that some vaccinations could be an economically attractive method for MAP control by dairy producers.

Risk factors for a high somatic cell count at the first milk recording in a large sample of UK dairy herds. By Madouasse et al., page 1873. Dairy cows usually undergo a 2-mo resting (dry) period between consecutive lactations, during which they are not milked. Milk somatic cell counts, a marker of inflammation, before and after the dry period were used to investigate the dynamics of intramammary infection during the dry period. Stage of lactation, parity, and milk yield before drying off were important in explaining individual cow performance, but only milk yield before drying off explained a significant part of the between-herd variability.

Chronic mastitis is associated with altered ovarian follicle development in dairy cattle. By Rahman et al., page 1885. The existence of a link between mastitis and fertility is a relatively new concept whose mechanisms are complex and largely undefined. Most data deal with the quantification of mastitis incidence at the herd level. The objective was to investigate whether the presence of mastitis affects the ovary. The presence of severe chronic mastitis was associated with an alteration of the follicle dynamics, a reduced vascular bed, and an increase of fibrotic tissue and reduced synthesis of a critical oocyte molecule. This is the first description of follicular development alteration during udder infection.

Effect of intrauterine dextrose or antibiotic therapy on reproductive performance of lactating dairy cows diagnosed with clinical endometritis. By Brick et al., page 1894. The study investigated the effect of intrauterine infusion of a hypertonic $50 \%$ dextrose solution (DEX) or subcutaneous antibiotic (ceftiofur crystalline free acid, CCFA) on the response to treatments (clinical cure and cow survival $14 \mathrm{~d}$ posttherapy) and reproductive performance of lactating dairy cows with and without clinical endometritis (CE). Administration of DEX or CCFA in conjunction with prostaglandin improved clinical cure $14 \mathrm{~d}$ posttherapy of cows with CE. Although the response to treatments and reproductive performance were similar among treatment groups, only the intrauterine infusion of DEX resulted in similar reproductive performance as cows without $\mathrm{CE}$.

Intramammary infections and teat canal colonization with coagulase-negative staphylococci after postmilking teat disinfection: Speciesspecific responses. By Quirk et al., page 1906. Coagulase-negative staphylococci (CNS) are a heterogeneous group of bacteria that remain a significant cause of intramammary infections (IMI) despite widespread adoption of postmilking teat disinfection by dairies. We hypothesized that teat disinfection would reduce the incidence of IMI by CNS but that the reduction would not be uniformly observed in all species. Findings supported the hypothesis as Staphylococcus chromogenes and Staphylococcus xylosus were the most prevalent CNS causing IMI without disinfection. However, with disinfection, the prevalence of Staph. xylosus IMI was reduced, whereas that of Staph. chromogenes IMI was unchanged. In aggregate, postmilking teat disinfection reduced teat canal colonization and IMI by CNS.

Minimum inhibitory concentrations of Staphylococcus aureus recovered from clinical and subclinical cases of bovine mastitis. By Ruegg et al., page 1913. Mastitis is the main cause of antimicrobial drug usage on dairy farms, and use of antimicrobials has been associated with antimicrobial resistance. Staphylococcus aureus is one of the most significant pathogens causing intramammary infections in dairy cattle worldwide. This study determined the prevalence of antimicrobial resistance of Staph. aureus $(\mathrm{n}=116)$ isolated from cases of clinical and subclinical mastitis that occurred on farms located in Wisconsin. Overall, $75 \%$ of Staph. aureus isolates were pansusceptible for the 12 antimicrobials, and $25 \%$ of isolates exhibited resistance for one or more antimicrobials. Only one isolate exhibited multidrug resistance. Of isolates, $19 \%$ were resistant to tetracycline and $14 \%$ to penicillin.

Herd-level association between antimicrobial use and antimicrobial resistance in bovine mastitis Staphylococcus aureus isolates on Canadian dairy farms. By Saini et al., page 1921. Concurrent data on antimicrobial use and resistance are needed to manage antimicrobial resistance in bacteria. The present study determined herd-level associations between antimicrobial use and resistance in Staphylococcus aureus isolated from milk samples on 89 Canadian dairy farms. Use of a penicillin-novobiocin combination for dry cow therapy was positively associated with penicillin and ampicillin resistance. Systemic administration of penicillin was also positively associated with penicillin resistance. Use of systemically administered florfenicol was positively associated with ampicillin re- 
sistance. Pirlimycin use for clinical mastitis treatment was positively associated with pirlimycin resistance. Regional differences in antimicrobial resistance were also observed.

Effect of dietary protein concentration on ammonia and greenhouse gas emitting potential of dairy manure. By Lee et al., page 1930. In two experiments, manure from cows fed high $(16.7 \%)$ and low $(14.8 \%$ ) crude protein (CP) diets was used to assess ammonia and greenhouse gas emissions from simulated short-term storage conditions and following soil application of fresh manure. The low CP diet substantially decreased ammonia emissions from manure during storage and when applied to soil. When manure application is based on plant nitrogen requirements, methane and carbon dioxide emissions may be increased for manure from low $\mathrm{CP}$ compared with high $\mathrm{CP}$ diets.

Effects of feeding increasing dietary levels of high oleic or regular sunflower or linseed oil on fatty acid profile of goat milk. By Martínez Marín et al., page 1942. The effects of increasing amounts of three plant oils in goat diets on the fatty acid (FA) profile of milk fat were studied. Linseed oil (LO) and regular sunflower oil (RSFO) linearly enriched milk fat in cis-9,trans-11-18:2 and trans-11-18:1; RSFO also increased linearly trans-10-18:1. High oleic sunflower oil (HOSFO) moderately raised trans-11-18:1 but not cis9,trans-11-18:2 in milk fat. Addition of LO decreased the ratio of n- 6 to $n-3$ polyunsaturated FA to about $70 \%$, whereas HOSFO and RSFO increased it. Addition of LO to alfalfa-concentrate goat diets was advantageous over addition of HOSFO or RSFO.

Rapeseed or linseed supplementation of grassbased diets: Effects on dairy performance of Holstein cows over 2 consecutive lactations. By Lerch et al., page 1956. Effects of linseed or rapeseed supplementation of grass-based diets on the performance of dairy cows were studied during 2 consecutive lactations, each comprising an indoor (winter, grass silage and hay-based diet) and an outdoor period (summer, pasture-based diet). The treatment effects were stable during the first indoor period and repeatable between the 2 outdoor periods and were similar to the effects observed in short-term studies (1 to $3 \mathrm{mo}$ ). Long-term linseed or rapeseed supplementation had minor effects on dairy cow production performance but induced a decrease in milk protein content during the indoor period.

Effects of grain, fructose, and histidine on ruminal $\mathrm{pH}$ and fermentation products during an induced acidosis protocol. By Golder et al., page 1971. Heifers were fed grain at $1.2 \%$ of body weight, to which $6 \mathrm{~g}$ of histidine was added and fructose at $0.4 \%$ of body weight was substituted for an equal amount of grain in a partial factorial design. Grain did not increase ruminal lactic acid concentrations. Fructose profoundly decreased rumen $\mathrm{pH}$ and increased total volatile fatty acids, butyrate, and lactic acid. Fructose feeding may increase the risk of subacute acidosis. Histidine did not directly affect rumen fermentation products but did increase histamine concentrations in the rumen.

Protein and energy intakes affected amino acid concentrations in plasma, muscle, and liver, and cell signaling in the liver of growing dairy calves. By Rius et al., page 1983. Feeding increasing amounts of protein and energy decreased plasma concentrations of Phe, Tyr, and Leu, but increased that of Thr and increased muscle to plasma ratios of Phe, Tyr, Leu, Ile, and Val, suggesting greater transport activity in response to greater supply of nutrients and body growth. Phosphorylation of protein kinase B was not affected by protein and energy intakes; however, treatment affected phosphorylation ratios of ribosomal protein S6 in the liver. Changes in plasma concentrations and tissue ratios of amino acids were consistent with enhanced growth rates.

A ring test of in vitro neutral detergent fiber digestibility: Analytical variability and sample ranking. By Hall and Mertens, page 1992. Laboratory measures of fiber digestion by ruminal microbes are commonly used to evaluate forage quality for use in formulating dairy cow diets, sometimes as numeric values, and sometimes to make qualitative changes in energy values. How precise are the digestibility measures we use? We found that laboratories very reliably ranked forage samples based on fiber digestibility, although the numeric values did vary by \pm 5 percentage units of digestibility. Thus, although the values are not very precise, the relative measures of fiber digestibility remain useful for accurately comparing the fiber digestibility of different forages for diet formulation.

Development and evaluation of equations in the Cornell Net Carbohydrate and Protein System to predict nitrogen excretion in lactating dairy cows. By Higgs et al., page 2004. Nitrogen (N) excretion on dairy farms is of concern because of the effect it has on water and air quality. The objective of this study was to evaluate and improve the ability of the Cornell Net Carbohydrate and Protein System (CNCPS) to predict fecal, urinary, and total manure $\mathrm{N}$ excretion in lactating dairy cows. Model predictions were improved by integrating alternative equations from the literature into the CNCPS framework, allowing nutritionists and farm advisors to characterize the potential environmental effect of rations at the time of formulation.

Rumen and milk odd- and branched-chain fatty acid proportions are minimally influenced by 
ruminal volatile fatty acid infusions. By French et al., page 2015. Rumen bacterial lipids contain odd- and branched-chain fatty acids (OBCFA) that are detected in milk fat. Therefore, milk OBCFA may serve as a tool to monitor rumen fermentation. In this study, acute intraruminal infusions of precursors for OBCFA resulted in few differences in rumen OBCFA. Supraphysiological intraruminal infusions of branched-chain volatile fatty acids markedly increased their concentration in blood without increasing corresponding milk OBCFA, suggesting that direct supply of these volatile fatty acids does not acutely influence synthesis of OBCFA by the mammary gland.

Effect of bromochloromethane on methane emission, rumen fermentation pattern, milk yield, and fatty acid profile in lactating dairy goats. By Abecia et al., page 202\%. The production of methane by ruminants represents an energetic inefficiency and significantly contributes to the emissions of greenhouse gases. However, no nutritional technology is fully available for the dairy sector to tackle this issue and little work has been published to conclusively associate a reduction of methane emissions with milk quality. This work used bromochloromethane as an antimethanogenic compound and observed a 33\% reduction in methane production, which was accompanied by a $36 \%$ increase in milk production but no detrimental effect on milk composition and fatty acid profile.

Chromium propionate enhances insulin sensitivity in growing cattle. By Spears et al., page $203 \%$. Chromium $(\mathrm{Cr})$ is a trace mineral that functions by potentiating the action of insulin. Chromium requirements of cattle have not been defined. This study determined the effects of dietary $\mathrm{Cr}(0,3,6$, or $9 \mathrm{mg}$ of $\mathrm{Cr} / \mathrm{d}$ ), in the form of $\mathrm{Cr}$ propionate, on insulin sensitivity in growing heifers. Chromium supplementation reduced insulin secretion and the ratio of insulin to glucose following glucose administration. Based on insulin sensitivity, Cr requirements of growing heifers fed Cr propionate do not exceed $3 \mathrm{mg}$ of $\mathrm{Cr} / \mathrm{d}$ or $0.47 \mathrm{mg}$ of $\mathrm{Cr} / \mathrm{kg}$ of dry matter.

Interaction of unsaturated fat or coconut oil with monensin in lactating dairy cows fed 12 times daily. I. Protozoal abundance, nutrient digestibility, and microbial protein flow to the omasum. By Reveneau et al., page 2046. Dietary fat potentially improves efficiency of microbial protein synthesis by inhibiting protozoa but can have negative effects on fiber digestibility or milk fat production, especially when combined with ionophores. We fed a control or $5 \%$ fat from animal-vegetable fat or coconut oil (a potent inhibitor of protozoa) factorialized without or with monensin to rumen-cannulated lactating Holsteins. Coconut oil depressed intake and neutral detergent fiber digestibility, and animal-vegetable fat depressed milk fat more when monensin was included. Under our conditions, any potential improvement in efficiency of dietary $\mathrm{N}$ usage from coconut oil with monensin was counteracted by decreased intake and milk fat production.

Interaction of unsaturated fat or coconut oil with monensin in lactating dairy cows fed 12 times daily. II. Fatty acid flow to the omasum and milk fatty acid profile. By Reveneau et al., page 2061. Dietary fat provides energy but also can inhibit fibrolytic bacteria and protozoa. Ruminal biohydrogenation influences accumulation of bioactive fatty acid isomers that depress milk fat synthesis. We fed a control or $5 \%$ fat from animal-vegetable fat or coconut oil (which is a potent inhibitor of protozoa) factorialized without or with monensin to rumen-cannulated lactating Holsteins. Feeding both fat sources increased trans-18:1 isomers in omasal digesta and milk fat. Biohydrogenation was decreased when coconut oil was fed, potentially directly or indirectly mediated through its suppression of protozoa.

Conservation characteristics of corn ear and stover ensiled with the addition of Lactobacillus plantarum MTD-1, Lactobacillus plantarum 30114 or Lactobacillus buchneri 11A44. By Lynch et al., page 2070. Because of the highly variable yield and nutritive value of forage corn produced under marginal growing conditions, conservation losses must be minimized. This study investigated the ability of 3 added bacteria to improve the efficiency of ensiling the ear and stover components of corn. No improvement in the ensiling efficiency of either component was observed in this study.

Use of a corn milling product in diets for dairy cows to alleviate milk fat depression. By Weiss, page 2081. Cows fed diets that are rapidly fermented in the rumen and that do not promote adequate chewing can produce milk with less than normal fat concentrations. Replacing mostly corn grain with a high-fiber, low-starch corn milling product alleviated milk fat depression caused by feeding a high-starch diet. When the corn milling product replaced both corn grain and forage, milk fat concentration remained depressed. High ratios of the dietary concentrations of starch to forage fiber was the best predictor of milk fat depression.

Short communication: Relationship of carbohydrate molecular spectroscopic features to carbohydrate nutrient profiles in co-products from bioethanol production. By Yu, page 2091. The objective of this study was to reveal carbohydrate molecular structure spectral profiles affected by bioethanol processing and quantify carbohydrate structure 
molecular spectral profiles in relation to carbohydrate digestive kinetics and nutritive value in the rumen and intestine in dairy cattle. The changes in carbohydrate molecular structures during the processing for bioethanol were highly associated with carbohydrate degradable subfractions.

Optimum multistage genomic selection in dairy cattle. By Börner et al., page 209\%. Arrays with a variable number of genetic markers serve as tools for genomic selection in dairy cattle. They differ both in their cost and achieved accuracies of estimated breeding values. Such marker sets could be applied either alone or in sequence, whereby genomic selection includes two stages. The sensitivity of genetic gain, as well as structure of a cooperative breeding program with a limited budget, was investigated under varying assumptions on cost and accuracies. The importance of multi-stage genomic selection was emphasized, especially for bull dams, but its economic practicability was questioned in terms of cost effectiveness.

Accuracy of genomic predictions of residual feed intake and 250-day body weight in growing heifers using 625,000 single nucleotide polymorphism markers. By Pryce et al., page 2108. The cost of feed in a dairy production system is about half of the total expense. Therefore, selection to improve feed conversion efficiency should lead to greater profitability of dairying. A measure of feed conversion efficiency is residual feed intake (RFI), which is the difference between actual and predicted feed intakes. However, measuring RFI on an industry-wide scale is not feasible because of the costs associated with measuring individual feed intakes. In this study, we demonstrated that a $625,000-$ SNP DNA panel explains a significant proportion of the genetic variation in RFI, enabling genomic selection for this important trait.

A comparison of partial least squares (PLS) and sparse PLS regressions in genomic selection in French dairy cattle. By Colombani et al., page 2120. Genomic selection relies on genomic estimated breeding values derived from dense marker information over the entire genome for the selection of young animals. Genomic estimated breeding value predictions need to be accurate for successful genomic selection. The aim of this study was to estimate the predictive abilities of partial least squares (PLS) and sparse PLS regressions compared with pedigree-based best linear unbiased predictors (BLUP) and genomic BLUP, which are currently used for the evaluation of French dairy cattle.

Makeup of the genetic correlation between milk production traits using genome-wide single nucleotide polymorphism information. By van Binsbergen et al., page 2132. Genome-wide association studies might be used to investigate the makeup of genetic correlations between traits. The objective of the present study was to investigate the potential of this approach by investigating the makeup of genetic correlations between milk production traits of dairy cows. Genetic correlations based on single nucleotide polymorphism or pedigree information differed, but little asymmetry of the genetic covariance was found, and most of the genetic variance was explained by many single nucleotide polymorphisms with a very small effect.

Incidences of and genetic parameters for mastitis, claw disorders, and common health traits recorded in dairy cattle contract herds. By Gernand et al., page 2144. Contract herds have been established with the primary goal of recording health traits that can be used for official genetic evaluations and for further applications such as genomic selection. A database was developed and recording of health disorders was done by farmers or veterinarians. Especially for clinical mastitis, relatively high incidences at the beginning of lactation in combination with antagonistic relationships with protein yield underline the necessity to implement breeding strategies that include direct selection on health disorders.

Short communication: Principal components and factor analytic models for test-day milk yield in Brazilian Holstein cattle. By Bignardi et al., page 215\%. The test-day model has been suggested as the method of choice for analysis of repeated measurements of milk production traits because it has the advantage of maximizing the use of all available information on genetic evaluation of dairy cattle. Different statistical models have been proposed to model test-day data. Currently, factor analysis and principal components provide powerful means to model multivariate covariance matrices, reducing the number of parameters to be estimated. The principal component and factor analytic models reduce computational requirements when comparable to a full rank analysis, which may facilitate analyses of larger data sets.

Short communication: A new bovine milkprotein variant: $\boldsymbol{\alpha}$-Lactalbumin variant D. $B y$ Visker et al., page 2165. An unknown protein variant of $\alpha$-lactalbumin was found in milk of a Holstein-Friesian cow. Sequencing genomic DNA of this cow showed a polymorphism that leads to histamine instead of glutamine at position 65 of the $\alpha$-lactalbumin protein. This is a new protein variant and should be called $\alpha$-lactalbumin protein variant $\mathrm{D}$. The amino acid change $65 \mathrm{Gln}>\mathrm{His}$ is not expected to affect protein function. Protein variant $\mathrm{D}$ of $\alpha$-lactalbumin does not seem to be frequent among dairy cattle, because its causing polymorphism was not detected in any of 156 bulls of 6 different dairy breeds. 
Technical note: Prediction of liveweight from linear conformation traits in dairy cattle. $B y B a-$ nos and Coffey, page 2170. Equations were developed to predict a cow's liveweight from her stature, chest width, body depth, and angularity classification score. This will enable the on-farm estimation of an otherwise difficult to record trait, which will facilitate management decision making. A different set of equations was developed to predict a bull's genetic merit for liveweight from his genetic evaluations for stature, chest width, body depth, and angularity. This is of particular importance to imported sires whose daughters' classification records are not available to phenotypically predict liveweight and calculate genetic evaluations.

Interaction between Lactococcus lactis and Lactococcus raffinolactis during growth in milk: Development of a new starter culture. By Kimoto-Nira et al., page 2176. To select a new mixed starter culture, 100 acid-producing bacterial strains were isolated from raw cow's milk. Mixed culture of Lactococcus lactis ssp. lactis no. 54 and Lactococcus raffinolactis no. 37 in milk stimulated greater acid production during fermentation than occurred with pure fermentation. Acid production of strain no. 37 was enhanced by metabolite from strain no. 54, whereas the converse effect was not observed. This study identifies a new commensal relationship between 2 lactococcal strains that are used for making dairy products.

Dairy cow behavior affects the availability of an automatic milking system. By Jacobs et al., page 2186. Little is known about how the interaction of cows with their environment and herdmates might affect the duration of time an automatic milking system (AMS) is available for milking. The objective was to assess behavior in and around the AMS unit and determine if any behaviors negatively affected the availability of the AMS. The results for each experimental group were conflicting, suggesting that social dynamics within each group may, at least partially, determine the availability and efficiency of an AMS unit.

Management and characteristics of recycled manure solids used for bedding in Midwest freestall dairy herds. By Husfeldt et al., page 2195.
Data were collected from 38 dairy farms in Wisconsin, Minnesota, Iowa, and South Dakota to characterize recycled manure solids (RMS) as a bedding material, observe bedding management practices, and document the methods of obtaining RMS. Methods of obtaining RMS for bedding included separation of digested manure, separation of raw manure, and separation of raw manure followed by mechanical drum-composting for 18 to 24 h. Moisture content of unused RMS was $72.4 \%$, with a $\mathrm{pH}$ of 9.16. Used digested solids contained fewer environmental streptococci than drum-composted and separated raw solids. Coliform counts were similar for all 3 bedding sources.

Characteristics of dairy calf ranches: Morbidity, mortality, antibiotic use practices, and biosecurity and biocontainment practices. By Walker et al., page 2204. Calf ranches are a lesser known yet increasingly utilized segment of the dairy industry specializing in rearing dairy calves. This work provides previously unreported estimates of morbidity, mortality, antibiotic use, and biosecurity and biocontainment practices on calf ranches rearing dairy heifer or heifer and bull calves. Calf ranchers and their advisors can use this information to assess the performance of an operation relative to the industry. Dairy producers, consultants, and veterinarians may find this information useful when deciding whether to use dairy calf ranches and when comparing calf ranch alternatives.

Potential gains in lifetime net merit from genomic testing of cows, heifers, and calves on commercial dairy farms. By Weigel et al., page 2215. Data were simulated for commercial dairy herds, and selection of animals was based on parent averages and predicted transmitting abilities with or without genomic testing. Gains in lifetime net merit due to genomic testing, minus prorated costs of genotyping, ranged from $\$ 28$ to $\$ 259$ for heifer calves with no pedigrees, $\$ 14$ to $\$ 121$ for heifer calves with known sires, and $\$ 7$ to $\$ 87$ for heifer calves with full pedigrees. Gains were much smaller for lactating cows. These results support that genomic testing can be a cost-effective tool for enhancing the genetic potential of replacement heifers on commercial farms. 